\title{
A Kronecker limit formula for totally real fields and arithmetic applications
}

\author{
Sheng-Chi Liu ${ }^{\top}$ and Riad Masri2 ${ }^{*}$
}

\author{
${ }^{*}$ Correspondence: \\ masri@math.tamu.edu \\ 2 Department of Mathematics, Texas \\ A\&M University, Mailstop 3368, \\ College Station, 77843-3368 TX, USA \\ Full list of author information is \\ available at the end of the article
}

\begin{abstract}
We establish a Kronecker limit formula for the zeta function $\zeta_{F}(s, A)$ of a wide ideal class $A$ of a totally real number field $F$ of degree $n$. This formula relates the constant term in the Laurent expansion of $\zeta_{F}(s, A)$ at $s=1$ to a toric integral of a $S L_{n}(\mathbb{Z})$-invariant function $\log G(Z)$ along a Heegner cycle in the symmetric space of $G L_{n}(\mathbb{R})$. We give several applications of this formula to algebraic number theory, including a relative class number formula for $H / F$ where $H$ is the Hilbert class field of $F$, and an analog of Kronecker's solution of Pell's equation for totally real multiquadratic fields. We also use a well-known conjecture from transcendence theory on algebraic independence of logarithms of algebraic numbers to study the transcendence of the toric integral of $\log G(Z)$. Explicit examples are given for each of these results.
\end{abstract}

Keywords: Maximal parabolic Eisenstein series; Heegner cycle; Kronecker limit formula; Transcendence

\section{Introduction and statement of results}

The celebrated Kronecker limit formula expresses the constant term in the Laurent expansion at $s=1$ of the Dedekind zeta function $\zeta_{K}(s, A)$ of an ideal class $A$ of an imaginary quadratic field $K$ in terms of the value of $\log |\eta(z)|$ at a Heegner point $\tau_{A}$ in the complex upper half-plane $\mathbb{H}$ where $\eta(z)$ is the Dedekind eta function

$$
\eta(z):=q^{1 / 24} \prod_{n=1}^{\infty}\left(1-q^{n}\right), \quad q:=e^{2 \pi i z} .
$$

There are many interesting applications of this formula to algebraic number theory, including relative class number formulae and Kronecker's "solution" to Pell's equation (see e.g. [8] Chapter II). Roughly speaking, to prove the Kronecker limit formula, one computes the constant term in the Laurent expansion at $s=1$ of the $S L_{2}$-Eisenstein series

$$
E(z, s):=\sum_{\gamma \in \Gamma_{\infty} \backslash S L_{2}(\mathbb{Z})} \operatorname{Im}(\gamma z)^{s}, \quad z \in \mathbb{H}, \quad \operatorname{Re}(s)>1
$$

then appeals to a classical identity of Dirichlet/Hecke relating $\zeta_{K}(s, A)$ to the value of $E(z, s)$ at the Heegner point $\tau_{A}$. Note that Hecke proved a similar limit formula for real quadratic fields by relating the ideal class zeta function to an integral of $E(z, s)$ over a Heegner cycle in $\mathbb{H}$.

(c) 2015 Liu and Masri. This is an Open Access article distributed under the terms of the Creative Commons Attribution License (http://creativecommons.org/licenses/by/2.0), which permits unrestricted use, distribution, and reproduction in any medium, provided the original work is properly cited. 
In the early 1980's, Bump and Goldfeld [2] proved a Kronecker limit formula for real cubic fields. This was based on an intriguing identity relating the integral of a minimal parabolic $S L_{3}$-Eisenstein series over a Heegner cycle to the Rankin/Selberg integral of a Hilbert modular Eisenstein series. Kudla [7] showed this identity was an instance of the so-called "basic identity" associated to a see-saw dual reductive pair. Efrat [4] later gave a Kronecker limit formula for non-real cubic fields by instead using the maximal parabolic $S L_{3}$-Eisenstein series. These results are just the tip of the iceberg. Indeed, as Bump and Goldfeld [2] remarked,

"Much work remains to be done in this direction, and one can only begin to see a whole new world of limit formulae emerging into view".

In this paper we will prove a Kronecker limit formula for the zeta function $\zeta_{F}(s, A)$ of a wide ideal class $A$ of a totally real number field $F$ of degree $n \geq 2$, thereby extending the limit formulae of Hecke and Bump/Goldfeld (see Theorem 1). This formula relates the constant term in the Laurent expansion of $\zeta_{F}(s, A)$ at $s=1$ to a toric integral of a $S L_{n}(\mathbb{Z})$ invariant function $\log G(Z)$ along a Heegner cycle in the symmetric space of $G L_{n}(\mathbb{R})$. We will give some applications of the limit formula to algebraic number theory, including a relative class number formula for $H / F$ where $H$ is the Hilbert class field of $F$, and an analog of Kronecker's solution of Pell's equation for totally real multiquadratic fields (see Theorems 2 and 3). We will also use a well-known conjecture from transcendence theory on algebraic independence of logarithms of algebraic numbers to study the transcendence of the toric integral of $\log G(Z)$ (see Corollary 1). Explicit examples are given in Section 2 for each of these results.

To prove the limit formula we will generalize the method of Efrat [4]. New difficulties arise when working with the maximal parabolic $S L_{n}$-Eisenstein series for arbitrary $n \geq 2$, though many of these may be overcome by appealing to work of Friedberg [5], Goldfeld [6] and Terras [10]. A key step in the proof is an identity relating $\zeta_{F}(s, A)$ to a toric integral of the maximal parabolic $S L_{n}$-Eisenstein series along a Heegner cycle in the symmetric space of $G L_{n}(\mathbb{R})$.

In order to state our results we fix the following notation. Let $\mathcal{H}^{n}=G L_{n}(\mathbb{R}) / O_{n}(\mathbb{R}) \mathbb{R}^{*}$ be the symmetric space of $G L_{n}(\mathbb{R})$. By the Iwasawa decomposition, each coset $Z \in \mathcal{H}^{n}$ has a unique representative of the form

$$
Z=\left(\begin{array}{ccccc}
1 & x_{1,2} & x_{1,3} & \ldots & x_{1, n} \\
1 & x_{2,3} & \ldots & x_{2, n} \\
& \ddots & & \vdots \\
& & 1 & x_{n-1, n} \\
& & & 1
\end{array}\right)\left(\begin{array}{lllll}
y_{1} y_{2} \cdots y_{n-1} & & & & \\
& y_{1} y_{2} \cdots y_{n-2} & & \\
& & & \ddots & \\
& & & y_{1} & \\
& & & & 1
\end{array}\right)
$$

where $x_{i, j} \in \mathbb{R}$ for $1 \leq i<j \leq n$ and $y_{i} \in \mathbb{R}_{+}$for $1 \leq i \leq n-1$. Left matrix multiplication induces an action of $G L_{n}(\mathbb{Z})$ on $\mathcal{H}^{n}$. For more details concerning these facts, see ([6] Section 1.2).

Let $P$ be the maximal parabolic subgroup of $S L_{n}(\mathbb{Z})$, which consists of those matrices with bottom row $(0, \ldots, 0,1)$. Define the maximal parabolic Eisenstein series

$$
E_{n}(Z, s):=\sum_{\gamma \in P \backslash S L_{n}(\mathbb{Z})} \operatorname{Det}(\gamma \cdot Z)^{s}, \quad \operatorname{Re}(s)>1
$$


where $\operatorname{Det}(\gamma \cdot Z)$ is the determinant of the unique representative of the coset $\gamma \cdot Z \in \mathcal{H}^{n}$ of the form (1) and $s \in \mathbb{C}$. Note that $E_{n}(Z, s)$ is well-defined since $\operatorname{Det}(p \cdot Z)=\operatorname{Det}(Z)$ for all $p \in P$. The completed Eisenstein series

$$
E_{n}^{*}(Z, s):=\pi^{-n s / 2} \Gamma(n s / 2) \zeta(n s) E_{n}(Z, s)
$$

satisfies the functional equation

$$
E_{n}^{*}(Z, s)=E_{n}^{*}\left(\left(Z^{T}\right)^{-1}, 1-s\right)
$$

and extends to a meromorphic function on $\mathbb{C}$ with simple poles at $s=0,1$.

Given $Z \in \mathcal{H}^{n}$, we may write

$$
Z Z^{T}=\left(\begin{array}{ll}
m & \mathbf{r}^{T} \\
\mathbf{r} & S
\end{array}\right)
$$

where

$$
\begin{aligned}
& m=\left(y_{1} y_{2}+\cdots y_{n-1}\right)^{2}+\left(x_{1,2} y_{1} y_{2} \cdots y_{n-2}\right)^{2}+\left(x_{1,3} y_{1} y_{2} \cdots y_{n-3}\right)^{2}+\cdots+x_{1, n}^{2}, \\
& \mathbf{r}=Z_{1}\left(\begin{array}{l}
x_{1,2} y_{1} y_{2} \cdots y_{n-2} \\
x_{1,3} y_{1} y_{2} \cdots y_{n-3} \\
\vdots \\
x_{1, n}
\end{array}\right)
\end{aligned}
$$

with

$$
Z_{1}=\left(\begin{array}{cccc}
1 & x_{2,3} & \ldots & x_{2, n} \\
& \ddots & & \vdots \\
& 1 & x_{n-1, n} \\
& & 1
\end{array}\right)\left(\begin{array}{llll}
y_{1} y_{2} \cdots y_{n-2} & & & \\
& & \ddots & \\
& & y_{1} & \\
& & & 1
\end{array}\right),
$$

and $S=Z_{1} Z_{1}^{T}$. Let

$$
\mathbf{q}=S^{-1} \mathbf{r}
$$

We will prove the following result.

Proposition 1. The Laurent expansion of $E_{n}^{*}(Z, s)$ at $s=1$ is given by

$$
E_{n}^{*}(Z, s)=\frac{2 / n}{s-1}+\gamma-\log (4 \pi)-\frac{2}{n} \log \left(y_{1} y_{2}^{2} \cdots y_{n-1}^{n-1}\right)-4 \log g(Z)+O(|s-1|),
$$

where $\gamma$ is Euler's constant and

$$
\begin{aligned}
g(Z):=\exp ( & \left.-\frac{\left(y_{1} y_{2}^{2} \cdots y_{n-1}^{n-1}\right)^{1 /(n-1)} E_{n-1}^{*}\left(Z_{1}, n /(n-1)\right)}{4}\right) \\
& \times \prod_{\substack{\left.\mathbf{b} \in \mathbb{Z}^{n-1} \\
\text { mod } \pm 1\right) \\
\mathbf{b} \neq \mathbf{0}}}\left|1-\exp \left(-2 \pi w^{1 / 2}\left(\mathbf{b}^{T} S^{-1} \mathbf{b}\right)^{1 / 2}+2 \pi i \mathbf{b}^{T} \mathbf{q}\right)\right| .
\end{aligned}
$$

Remark 1. The function $g(Z)$ is a $G L_{n}$ analog of $|\eta(z)|$ which generalizes the $G L_{3}$ analog defined in ([4] p. 175). 
Let $F$ be a totally real number field of degree $n$ and $U$ be the group of units of $F$. Let $A$ be a wide ideal class of $F$ and define the ideal class zeta function

$$
\zeta_{F}(s, A):=\sum_{\substack{\mathfrak{A} \in A \\ \mathfrak{A} \neq 0}} \frac{1}{N(\mathfrak{A})^{s}}, \quad \operatorname{Re}(s)>1
$$

where $N(\mathfrak{A})$ is the norm. The completed zeta function is defined by

$$
\zeta_{F}^{*}(s, A):=\pi^{-n s / 2} \Gamma(s / 2)^{n} D_{F}^{s / 2} \zeta_{F}(s, A),
$$

where $D_{F}$ is the discriminant of $F$. The function $\zeta_{F}^{*}(s, A)$ satisfies the functional equation

$$
\zeta_{F}^{*}(s, A)=\zeta_{F}^{*}(1-s, A)
$$

and extends to a meromorphic function on $\mathbb{C}$ with a simple pole at $s=1$. We will calculate the constant term in the Laurent expansion of $\zeta_{F}^{*}(s, A)$ at $s=1$ by relating $\zeta_{F}^{*}(s, A)$ to a toric integral of $E_{n}^{*}(Z, s)$ along a Heegner cycle in $\mathcal{H}^{n}$ associated to $A$ and appealing to Proposition 1.

Fix an ideal $\mathfrak{B} \in A^{-1}$. Let $\alpha_{1}, \alpha_{2}, \ldots, \alpha_{n}$ be a $\mathbb{Z}$-basis for $\mathfrak{B}$ and $\alpha_{1}^{(i)}, \alpha_{2}^{(i)}, \ldots, \alpha_{n}^{(i)}$ for $i=1,2, \ldots, n$ denote their images under the real embeddings of $F$. Define the matrix

$$
M_{\mathfrak{B}}(\mathbf{t}):=\left(\begin{array}{ccccc}
\alpha_{1}^{(1)} t_{1} & \alpha_{1}^{(2)} t_{2} & \cdots & \alpha_{1}^{(n-1)} t_{n-1} & \alpha_{1}^{(n)}\left(t_{1} t_{2} \cdots t_{n-1}\right)^{-1} \\
\alpha_{2}^{(1)} t_{1} & \alpha_{2}^{(2)} t_{2} & \cdots & \alpha_{2}^{(n-1)} t_{n-1} & \alpha_{2}^{(n)}\left(t_{1} t_{2} \cdots t_{n-1}\right)^{-1} \\
\vdots & \vdots & & \vdots & \vdots \\
\alpha_{n}^{(1)} t_{1} & \alpha_{n}^{(2)} t_{2} & \cdots & \alpha_{n}^{(n-1)} t_{n-1} & \alpha_{n}^{(n)}\left(t_{1} t_{2} \cdots t_{n-1}\right)^{-1}
\end{array}\right)
$$

where $\mathbf{t}=\left(t_{1}, t_{2}, \ldots, t_{n-1}\right) \in \mathbb{R}_{+}^{n-1}$, and let

$$
Q_{\mathfrak{B}}(\mathbf{t}):=M_{\mathfrak{B}}(\mathbf{t}) M_{\mathfrak{B}}(\mathbf{t})^{T} .
$$

The positive definite, symmetric matrix $Q_{\mathfrak{B}}(\mathbf{t})$ may be written as

$$
Q_{\mathfrak{B}}(\mathbf{t})=\operatorname{Det}\left(Q_{\mathfrak{B}}(\mathbf{t})\right)^{1 / n}\left(y_{1}(\mathbf{t})^{n-1} y_{2}(\mathbf{t})^{n-2} \cdots y_{n-1}(\mathbf{t})\right)^{-2 / n} \tau_{\mathfrak{B}}(\mathbf{t}) \tau_{\mathfrak{B}}(\mathbf{t})^{T},
$$

where

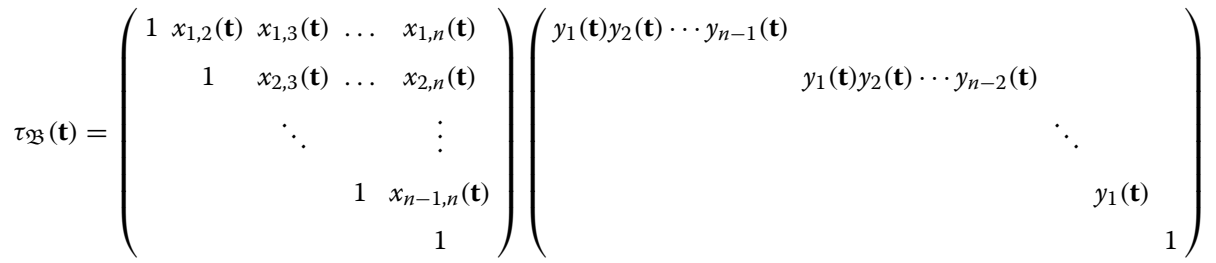

is in $\mathcal{H}^{n}$. Here we have suppressed the dependence of the variables $x_{i, j}(\mathbf{t})$ and $y_{i}(\mathbf{t})$ on $\mathfrak{B}$ and the $\mathbb{Z}$-basis $\alpha_{1}, \alpha_{2}, \ldots, \alpha_{n}$.

Given a unit $\varepsilon \in U$, let $\varepsilon_{1}, \varepsilon_{2}, \ldots, \varepsilon_{n}$ denote the images of $\varepsilon$ under the real embeddings of $F$. There is an action of the unit group $U$ on $\mathbb{R}_{+}^{n-1}$ given by

$$
\begin{aligned}
\varepsilon: & \mathbb{R}_{+}^{n-1} \longrightarrow \mathbb{R}_{+}^{n-1}, \\
& \left(t_{1}, t_{2}, \ldots, t_{n-1}\right) \longmapsto\left(\left|\varepsilon_{1}\right| t_{1},\left|\varepsilon_{2}\right| t_{2}, \ldots,\left|\varepsilon_{n-1}\right| t_{n-1}\right) .
\end{aligned}
$$

Let $\mathbb{R}_{+}^{n-1} / U$ denote a fundamental domain for this action. Then $\left\{\tau_{\mathfrak{B}}(\mathbf{t}): \mathbf{t} \in \mathbb{R}_{+}^{n-1} / U\right\}$ defines a Heegner cycle in $\mathcal{H}^{n}$.

We will establish the following identity. 
Proposition 2. Let $F$ be a totally real number field of degree $n$ and $A$ be a wide ideal class of F. Then

$$
\zeta_{F}^{*}(s, A)=n 2^{n-1} \int_{\mathbb{R}_{+}^{n-1} / U} \ldots \int_{n} E_{n}^{*}\left(\tau_{\mathfrak{B}}(\mathbf{t}), s\right) \frac{d t_{1}}{t_{1}} \cdots \frac{d t_{n-1}}{t_{n-1}}
$$

where $\left\{\tau_{\mathfrak{B}}(\mathbf{t}): \mathbf{t} \in \mathbb{R}_{+}^{n-1} / U\right\}$ is the Heegner cycle associated to $\mathfrak{B} \in A^{-1}$.

Remark 2. Although the Heegner cycle $\left\{\tau_{\mathfrak{B}}(\mathbf{t}): \mathbf{t} \in \mathbb{R}_{+}^{n-1} / U\right\}$ depends on the ideal $\mathfrak{B} \in A^{-1}$ and the choice of integral basis for $\mathfrak{B}$, the integral on the right hand side of (2) depends only on the ideal class $A$.

Remark 3. One can identify $\mathbb{R}_{+}^{n-1}$ with

$$
T^{n}=\left\{\left(t_{1}, t_{2}, \cdots, t_{n-1}, t_{n}\right) \in \mathbb{R}_{+}^{n}: \prod_{i=1}^{n} t_{i}=1\right\} .
$$

Using this identification, (2) can be written as an integral over $T^{n} / U$ with respect to the $U$-invariant measure on $T^{n}$.

By combining Propositions 1 and 2, we will obtain the following Kronecker limit formula for the zeta function of a wide ideal class of a totally real field.

Theorem 1. Let $F$ be a totally real number field of degree $n$ and $A$ be a wide ideal class of F. Then

$$
\begin{aligned}
& \lim _{s \rightarrow 1}\left[\zeta_{F}^{*}(s, A)-\frac{2^{n} \operatorname{vol}\left(\mathbb{R}_{+}^{n-1} / U\right)}{s-1}\right]= \\
& n 2^{n-1} \gamma \operatorname{vol}\left(\mathbb{R}_{+}^{n-1} / U\right)-n 2^{n-1} \int_{\mathbb{R}_{+}^{n-1} / U} \ldots \int \log G\left(\tau_{\mathfrak{B}}(\mathbf{t})\right) \frac{d t_{1}}{t_{1}} \cdots \frac{d t_{n-1}}{t_{n-1}}
\end{aligned}
$$

where

$$
G(Z):=4 \pi\left(y_{1} y_{2}^{2} \cdots y_{n-1}^{n-1}\right)^{2 / n} g(Z)^{4}
$$

and $\left\{\tau_{\mathfrak{B}}(\mathbf{t}): \mathbf{t} \in \mathbb{R}_{+}^{n-1} / U\right\}$ is the Heegner cycle associated to $\mathfrak{B} \in A^{-1}$.

Proof. We may write the Laurent expansion in Proposition 1 as

$$
E_{n}^{*}(Z, s)=\frac{2 / n}{s-1}+\gamma-\log G(Z)+O(|s-1|)
$$

where $G(Z)$ is defined as in the statement of the theorem. In particular, this shows that $G(Z)$ is $S L_{n}(\mathbb{Z})$-invariant. Inserting (3) into the integral on the right hand side of (2) immediately yields the result.

Remark 4. By Remark 2, the integral appearing in Theorem 1 depends only on the ideal class $A$ of $F$. In some of the applications which follow, it will be convenient to denote this integral by 


$$
\rho_{n}(A):=\int_{\mathbb{R}_{+}^{n-1} / U} \ldots \int_{\log } \log \left(\tau_{\mathfrak{B}}(\mathbf{t})\right) \frac{d t_{1}}{t_{1}} \cdots \frac{d t_{n-1}}{t_{n-1}} .
$$

We now give some applications of Theorem 1 to algebraic number theory in the spirit of Siegel ([8] Chapter II). Given a number field $K$, let $\mathrm{CL}(K)$ be the wide ideal class group, $h_{K}$ be the class number, $R_{K}$ be the regulator, $w_{K}$ be the number of roots of unity, and $D_{K}$ be the absolute value of the discriminant. We will prove the following relative class number formula for $H / F$ where $H$ is the Hilbert class field of $F$.

Theorem 2. Let $F$ be a totally real number field of degree $n$ and $H$ be the Hilbert class field of $F$. Write the ideal class group of $F$ as

$$
\mathrm{CL}(F)=\left\{\left[\mathfrak{A}_{1}\right]=\left[\mathcal{O}_{F}\right],\left[\mathfrak{A}_{2}\right], \ldots,\left[\mathfrak{A}_{h_{F}}\right]\right\} .
$$

Then

$$
\frac{(-1)^{h_{F}-1} 2^{h_{F}-1}}{n^{h_{F}-1}} \frac{h_{H}}{h_{F}} \frac{R_{H}}{R_{F}}=\operatorname{Det}\left(\int_{\mathbb{R}_{+}^{n-1} / U} \ldots \int_{U} \log \left(\frac{G\left(\tau_{\mathfrak{A}_{\ell}^{-1} \mathfrak{A}_{k}}(\mathbf{t})\right)}{G\left(\tau_{\mathfrak{A}_{\ell}}(\mathbf{t})\right)}\right) \frac{d t_{1}}{t_{1}} \ldots \frac{d t_{n-1}}{t_{n-1}}\right)_{k, \ell}
$$

where $1 \leq k, \ell \leq h_{F}-1$.

We will also prove the following analog of Kronecker's solution of Pell's equation for totally real multiquadratic fields. A result of this type for real quadratic fields is given in ([8] p. 97, Proposition 13).

Theorem 3. Let $F$ be a totally real abelian number field with $\mathrm{Gal}(F / \mathbb{Q}) \cong(\mathbb{Z} / 2 \mathbb{Z})^{\ell}$. Let $E$ be an unramified real quadratic extension of $F$ with $\operatorname{Gal}(E / \mathbb{Q}) \cong(\mathbb{Z} / 2 \mathbb{Z})^{\ell+1}$ and $\chi_{E / F}$ be the genus character of $F$ associated to $E / F$ by class field theory. Then

$$
\sum_{A \in \mathrm{CL}(F)} \chi_{E / F}(A) \rho_{2^{\ell}}(A)=-\frac{D_{F}^{1 / 2}}{2^{\ell-1}} \prod_{i=1}^{2^{\ell}} \frac{\log \left(\varepsilon_{i}\right) h_{i}}{\sqrt{\Delta_{i}}},
$$

where $\Delta_{i}>0$ for $1 \leq i \leq 2^{\ell}$ are the discriminants of the quadratic subfields $K_{i}$ of $E$ which are not contained in $F, \varepsilon_{i}$ is the fundamental unit of $K_{i}$, and $h_{i}$ is the class number of $K_{i}$.

Next, let

$$
\mathcal{L}:=\left\{\log (\alpha): \alpha \in \overline{\mathbb{Q}}^{*}\right\}
$$

be the set of logarithms of algebraic numbers. The following is a well-known conjecture on algebraic independence from transcendence theory (see e.g. [11] Conjecture 1.15).

Conjecture 1 (Algebraic Independence of Logarithms). If $\lambda_{1}, \lambda_{2}, \ldots, \lambda_{k}$ are $\mathbb{Q}$-linearly independent elements of $\mathcal{L}$, then $\lambda_{1}, \lambda_{2}, \ldots, \lambda_{k}$ are algebraically independent over $\mathbb{Q}$.

Assuming Conjecture 1, we will prove the following result. 
Corollary 1. Let notation and assumptions be as in Theorem 3. If Conjecture 1 is true, then

$$
\sum_{A \in \mathrm{CL}(F)} \chi_{E / F}(A) \rho_{2^{\ell}}(A)
$$

is transcendental.

Organization. The paper is organized as follows. In Section 2 we give some explicit examples of Theorems 2 and 3. In Sections 3 and 4 we prove Propositions 1 and 2, resp. In Sections 5, 6 and 7, we prove Theorems 2, 3 and Corollary 1, resp.

\section{Examples}

In this section we give some explicit examples of Theorems 2 and 3.

Example 1. Consider the tower of fields

$$
\mathbb{Q} \subset F \subset F_{\text {gen }} \subset H
$$

where $F=\mathbb{Q}(\sqrt{5}, \sqrt{3 \cdot 29}), F_{\text {gen }}=\mathbb{Q}(\sqrt{3}, \sqrt{5}, \sqrt{29}, \sqrt{7+2 \sqrt{5}})$ is the genus field of $F$, i.e., the maximal unramified extension of $F$ which is abelian over $\mathbb{Q}$, and $H$ is the Hilbert class field of $F$. The genus field $F_{\text {gen }}$ was determined in ([12] Example 2.2 (1)). Note that because $F$ has class number $h_{F}=4$ and $F_{\text {gen }} / F$ is an unramified abelian extension of degree $4=h_{F}=[H: F]$, the genus field $F_{\text {gen }}$ is actually the Hilbert class field $H$. Now, the ideal class group of $F$ is $C L(F) \cong \mathbb{Z} / 2 \mathbb{Z} \oplus \mathbb{Z} / 2 \mathbb{Z}$. A set of representatives of the (nontrivial) ideal classes $\operatorname{CL}(F)=\left\{\left[\mathfrak{A}_{1}\right]=\left[\mathcal{O}_{F}\right],\left[\mathfrak{A}_{2}\right],\left[\mathfrak{A}_{3}\right],\left[\mathfrak{A}_{4}\right]\right\}$ is given by

$$
\begin{aligned}
& \mathfrak{A}_{2}=\mathfrak{A}_{2}^{-1}=\left\langle 19,\left(-\frac{9}{2} \sqrt{3 \cdot 29}+3\right) \sqrt{5}-\frac{5}{2} \sqrt{3 \cdot 29}+198\right\rangle \\
& \mathfrak{A}_{3}=\mathfrak{A}_{3}^{-1}=\left\langle 31,\left(-\frac{1}{2} \sqrt{3 \cdot 29}+13\right) \sqrt{5}-\frac{25}{2} \sqrt{3 \cdot 29}+15\right\rangle \\
& \mathfrak{A}_{4}=\mathfrak{A}_{4}^{-1}=\left\langle 3,\left(-\frac{1}{2} \sqrt{3 \cdot 29}-\frac{3}{2}\right) \sqrt{5}+\frac{3}{2} \sqrt{3 \cdot 29}+\frac{45}{2}\right\rangle .
\end{aligned}
$$

Applying Theorem 2 to these particular fields yields the following formula for the class number of $H=\mathbb{Q}(\sqrt{3}, \sqrt{5}, \sqrt{29}, \sqrt{7+2 \sqrt{5}})$,

$$
h_{H}=-32 \frac{R_{F}}{R_{H}} \operatorname{Det}\left(\begin{array}{lll}
u_{11} & u_{12} & u_{13} \\
u_{21} & u_{22} & u_{23} \\
u_{31} & u_{32} & u_{33}
\end{array}\right)
$$

where

$$
u_{k \ell}=\iiint_{\mathbb{R}_{+}^{3} / U} \log \left(\frac{G\left(\tau_{\mathfrak{A}_{\ell}^{-1} \mathfrak{A}_{k}}(\mathbf{t})\right)}{G\left(\tau_{\mathfrak{A}_{\ell}}(\mathbf{t})\right)}\right) \frac{d t_{1}}{t_{1}} \frac{d t_{2}}{t_{2}} \frac{d t_{3}}{t_{3}}, \quad 1 \leq k, \ell \leq 3 .
$$

In particular, if $\Delta$ denotes the determinant on the right hand side of (4), we have

$$
\frac{R_{F}}{R_{H}} \Delta \in \mathbb{Q} .
$$

Remark 5. A formula for the ratio of regulators $R_{H} / R_{F}$ may be deduced from ([3] Theorem 1). 
Example 2. Consider the tower of fields

$$
\mathbb{Q} \subset F \subset E \subset F_{\text {gen }}=H
$$

where $F$ and $F_{\text {gen }}=H$ are as in Example 1 and $E=\mathbb{Q}(\sqrt{3}, \sqrt{5}, \sqrt{29})$. Then $F$ is a real biquadratic field and $E$ is an unramified real quadratic extension of $F$ with $\operatorname{Gal}(E / \mathbb{Q}) \cong$ $(\mathbb{Z} / 2 \mathbb{Z})^{3}$. Recall the set of representatives $\operatorname{CL}(F)=\left\{\left[\mathfrak{A}_{1}\right]=\left[\mathcal{O}_{F}\right],\left[\mathfrak{A}_{2}\right],\left[\mathfrak{A}_{3}\right],\left[\mathfrak{A}_{4}\right]\right\}$ for the ideal class group of $F$ that were given in Example 1. Since the ideal $\mathfrak{A}_{4}$ becomes principal in $E$ (it is generated by $\sqrt{3}$ ), the genus character $\chi_{E / F}: \operatorname{CL}(F) \rightarrow\{ \pm 1\}$ is given by $\chi_{E / F}\left(\left[\mathfrak{A}_{1}\right]\right)=\chi_{E / F}\left(\left[\mathfrak{A}_{4}\right]\right)=1$ and $\chi_{E / F}\left(\left[\mathfrak{A}_{2}\right]\right)=\chi_{E / F}\left(\left[\mathfrak{A}_{3}\right]\right)=-1$. The discriminant of $F$ is $D_{F}=3027600=2^{4} \cdot 3^{2} \cdot 5^{2} \cdot 29^{2}$. The quadratic subfields of $E$ not contained in $F$ are $K_{1}=\mathbb{Q}(\sqrt{3}), K_{2}=\mathbb{Q}(\sqrt{29}), K_{3}=\mathbb{Q}(\sqrt{3 \cdot 5})$ and $K_{4}=\mathbb{Q}(\sqrt{5 \cdot 29})$. The corresponding class numbers and discriminants are $h_{1}=1, h_{2}=1, h_{3}=2, h_{4}=4$, and $\Delta_{1}=12, \Delta_{2}=29, \Delta_{3}=60, \Delta_{4}=145$, resp. The fundamental units are $\varepsilon_{1}=2+\sqrt{3}$, $\varepsilon_{2}=(5+\sqrt{29}) / 2, \varepsilon_{3}=4+\sqrt{15}$ and $\varepsilon_{4}=12+\sqrt{145}$. Applying Theorem 3 to these particular fields yields the identity

$$
\begin{aligned}
\iiint_{\mathbb{R}_{+}^{3} / U} \log & \left(\frac{G\left(\tau_{\mathfrak{A}_{1}^{-1}}(\mathbf{t})\right) G\left(\tau_{\mathfrak{A}_{4}^{-1}}(\mathbf{t})\right)}{G\left(\tau_{\mathfrak{A}_{2}^{-1}}(\mathbf{t})\right) G\left(\tau_{\mathfrak{A}_{3}^{-1}}(\mathbf{t})\right)}\right) \frac{d t_{1}}{t_{1}} \frac{d t_{2}}{t_{2}} \frac{d t_{3}}{t_{3}}= \\
& -4 \log (2+\sqrt{3}) \log ((5+\sqrt{29}) / 2) \log (4+\sqrt{15}) \log (12+\sqrt{145}) .
\end{aligned}
$$

Moreover, by Corollary 1 (which assumes Conjecture 1 ) the number

$$
\iiint_{\mathbb{R}_{+}^{3} / U} \log \left(\frac{G\left(\tau_{\mathfrak{A}_{1}^{-1}}(\mathbf{t})\right) G\left(\tau_{\mathfrak{A}_{4}^{-1}}(\mathbf{t})\right)}{G\left(\tau_{\mathfrak{A}_{2}^{-1}}(\mathbf{t})\right) G\left(\tau_{\mathfrak{A}_{3}^{-1}}(\mathbf{t})\right)}\right) \frac{d t_{1}}{t_{1}} \frac{d t_{2}}{t_{2}} \frac{d t_{3}}{t_{3}}
$$

is transcendental.

\section{Maximal parabolic Eisenstein series on $S L_{n}(\mathbb{Z})$}

In this section we compute the Laurent expansion at $s=1$ of the maximal parabolic Eisenstein series on $S L_{n}(\mathbb{Z})$ and thus prove Proposition 1 . We follow closely the work of Efrat [4], Friedberg [5], Goldfeld [6] and Terras [10].

For convenience, we recall the setup from Section 1 . Let $\mathcal{H}^{n}=G L_{n}(\mathbb{R}) / O_{n}(\mathbb{R}) \mathbb{R}^{*}$ be the symmetric space of $G L_{n}(\mathbb{R})$. By the Iwasawa decomposition, each coset $Z \in \mathcal{H}^{n}$ has a unique representative of the form

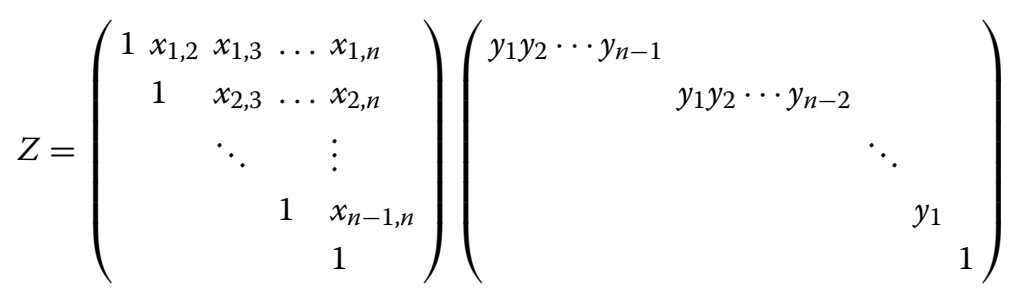

where $x_{i, j} \in \mathbb{R}$ for $1 \leq i<j \leq n$ and $y_{i} \in \mathbb{R}_{+}$for $1 \leq i \leq n-1$. Left matrix multiplication induces an action of $G L_{n}(\mathbb{Z})$ on $\mathcal{H}^{n}$.

Let $P$ be the maximal parabolic subgroup of $S L_{n}(\mathbb{Z})$, which consists of those matrices with bottom row $(0, \ldots, 0,1)$. Define the maximal parabolic Eisenstein series 


$$
E_{n}(Z, s):=\sum_{\gamma \in P \backslash S L_{n}(\mathbb{Z})} \operatorname{Det}(\gamma \cdot Z)^{s}, \quad \operatorname{Re}(s)>1
$$

where $\operatorname{Det}(\gamma \cdot Z)$ is the determinant of the unique representative of the $\operatorname{coset} \gamma \cdot Z \in \mathcal{H}^{n}$ of the form (5) and $s \in \mathbb{C}$. The completed Eisenstein series

$$
E_{n}^{*}(Z, s):=\pi^{-n s / 2} \Gamma(n s / 2) \zeta(n s) E_{n}(Z, s)
$$

satisfies the functional equation

$$
E_{n}^{*}(Z, s)=E_{n}^{*}\left(\left(Z^{T}\right)^{-1}, 1-s\right)
$$

and extends to a meromorphic function on $\mathbb{C}$ with simple poles at $s=0,1$.

Define

$$
Q=Q Z:=Z Z^{T}
$$

and let

$$
Q[\mathbf{a}]:=\mathbf{a}^{T} Q \mathbf{a} \quad \text { for } \quad \mathbf{a}=\left(\begin{array}{l}
a_{1} \\
a_{2} \\
\vdots \\
a_{n}
\end{array}\right) .
$$

Then one has the identity (see e.g. [6] p. 308-309, eq. (10.7.4))

$$
\zeta(n s) E_{n}(Z, s)=\operatorname{Det}(Z)^{s} \zeta(n s / 2, Q),
$$

where

$$
\zeta(s, Q):=\sum_{\substack{\mathbf{a} \in \mathbb{Z}^{n} \\ \mathbf{a} \neq \mathbf{0}}} Q[\mathbf{a}]^{-s}, \quad \operatorname{Re}(s)>\frac{n}{2}
$$

is the Epstein zeta function of $Q$. In particular, since

$$
\operatorname{Det}(Z)=y_{1}^{n-1} y_{2}^{n-2} \cdots y_{n-1}
$$

we have

$$
E_{n}^{*}(Z, s)=\pi^{-n s / 2} \Gamma(n s / 2)\left(y_{1}^{n-1} y_{2}^{n-2} \cdots y_{n-1}\right)^{s} \sum_{\substack{\mathbf{a} \in \mathbb{Z}^{n} \\ \mathbf{a} \neq \mathbf{0}}} Q[\mathbf{a}]^{-n s / 2} .
$$

We now compute the Laurent expansion of $E_{n}^{*}(Z, s)$ at $s=1$ by splitting the sum in (7) into terms with $a_{1}=0$ and terms with $a_{1} \neq 0$.

Write

$$
Q=\left(\begin{array}{ll}
m & \mathbf{r}^{T} \\
\mathbf{r} & S
\end{array}\right)
$$

where

$$
\begin{aligned}
& m=\left(y_{1} y_{2}+\cdots y_{n-1}\right)^{2}+\left(x_{1,2} y_{1} y_{2} \cdots y_{n-2}\right)^{2}+\left(x_{1,3} y_{1} y_{2} \cdots y_{n-3}\right)^{2}+\cdots+x_{1, n}^{2}, \\
& \mathbf{r}=Z_{1}\left(\begin{array}{c}
x_{1,2} y_{1} y_{2} \cdots y_{n-2} \\
x_{1,3} y_{1} y_{2} \cdots y_{n-3} \\
\vdots \\
x_{1, n}
\end{array}\right)
\end{aligned}
$$


with

$$
Z_{1}=\left(\begin{array}{cccc}
1 & x_{2,3} & \ldots & x_{2, n} \\
& \ddots & & \vdots \\
& 1 & x_{n-1, n} \\
& & 1
\end{array}\right)\left(\begin{array}{llll}
y_{1} y_{2} \cdots y_{n-2} & & \\
& & \ddots & \\
& & y_{1} & \\
& & & 1
\end{array}\right),
$$

and $S=Z_{1} Z_{1}^{T}$. Also, write $\mathbf{a}=\left(\begin{array}{c}a_{1} \\ \mathbf{b}\end{array}\right)$ where

$$
\mathbf{b}=\left(\begin{array}{l}
a_{2} \\
a_{3} \\
\vdots \\
a_{n}
\end{array}\right)
$$

If $a_{1}=0$ then $Q[\mathbf{a}]=S[\mathbf{b}]$, hence the contribution of the terms with $a_{1}=0$ in (7) is

$$
\begin{gathered}
\pi^{-n s / 2} \Gamma(n s / 2)\left(y_{1}^{n-1} y_{2}^{n-2} \cdots y_{n-1}\right)^{s} \sum_{\substack{\mathbf{b} \in \mathbb{Z}^{n-1} \\
\mathbf{b} \neq \mathbf{0}}} S[\mathbf{b}]^{-n s / 2} \\
=\left(y_{1} y_{2}^{2} \cdots y_{n-1}^{n-1}\right)^{s /(n-1)} E_{n-1}^{*}\left(Z_{1}, n /(n-1) s\right) .
\end{gathered}
$$

Let $s=1$ to get

$$
\left(y_{1} y_{2}^{2} \cdots y_{n-1}^{n-1}\right)^{1 /(n-1)} E_{n-1}^{*}\left(Z_{1}, n /(n-1)\right) .
$$

Next, suppose that $a_{1} \neq 0$. We need to analyze

$$
\pi^{-n s / 2} \Gamma(n s / 2)\left(y_{1}^{n-1} y_{2}^{n-2} \cdots y_{n-1}\right)^{s} \sum_{\substack{\mathbf{a} \in \mathbb{Z}^{n} \\ a_{1} \neq 0}} Q[\mathbf{a}]^{-n s / 2} .
$$

Let $\mathbf{q}=S^{-1} \mathbf{r}$ and $w=m-\mathbf{q}^{T} S \mathbf{q}$. Then

$$
Q=\left(\begin{array}{ll}
m & \mathbf{r}^{T} \\
\mathbf{r} & S
\end{array}\right)=\left(\begin{array}{cc}
1 & \mathbf{q}^{T} \\
& \mathrm{I}_{n-1}
\end{array}\right)\left(\begin{array}{ll}
w & \\
& S
\end{array}\right)\left(\begin{array}{ll}
1 & \\
\mathbf{q} & \mathrm{I}_{n-1}
\end{array}\right),
$$

so that

$$
Q[\mathbf{a}]=w\left[a_{1}\right]+S\left[\mathbf{q} a_{1}+\mathbf{b}\right] .
$$

Hence (9) may be written as

$$
\pi^{-n s / 2} \Gamma(n s / 2)\left(y_{1}^{n-1} y_{2}^{n-2} \cdots y_{n-1}\right)^{s} \sum_{\substack{a_{1} \in \mathbb{Z} \\ a_{1} \neq 0}} \sum_{\mathbf{b} \in \mathbb{Z}^{n-1}}\left(w\left[a_{1}\right]+S\left[\mathbf{q} a_{1}+\mathbf{b}\right]\right)^{-n s / 2} .
$$

We wish to apply the Poisson summation formula to the sum over $\mathbf{b} \in \mathbb{Z}^{n-1}$ in (10). Define

$$
f(\mathbf{x}, s):=\left(w\left[a_{1}\right]+S\left[\mathbf{q} a_{1}+\mathbf{x}\right]\right)^{-n s / 2}, \quad \mathbf{x} \in \mathbb{R}^{n-1} .
$$

The Fourier transform is given by

$$
\widehat{f}(\mathbf{y}, s)=\int_{\mathbb{R}^{n-1}}\left(w\left[a_{1}\right]+S\left[\mathbf{q} a_{1}+\mathbf{x}\right]\right)^{-n s / 2} \exp \left(-2 \pi i \mathbf{y}^{T} \mathbf{x}\right) d \mathbf{x} .
$$

Write $S=W^{T} W$ and make the change of variables $\mathbf{u}=\left(w\left[a_{1}\right]\right)^{-1 / 2} W\left(\mathbf{q} a_{1}+\mathbf{x}\right)$. Then

$$
\mathbf{x}=\left(w\left[a_{1}\right]\right)^{1 / 2} W^{-1} \mathbf{u}-\mathbf{q} a_{1}
$$


and

$$
d \mathbf{x}=\left(w\left[a_{1}\right]\right)^{(n-1) / 2} \operatorname{Det}(W)^{-1} d \mathbf{u}=\left(w\left[a_{1}\right]\right)^{(n-1) / 2} \operatorname{Det}(S)^{-1 / 2} d \mathbf{u},
$$

so that

$\widehat{f}(\mathbf{y}, s)=\left(w\left[a_{1}\right]\right)^{\frac{n-1}{2}-\frac{n s}{2}} \operatorname{Det}(S)^{-1 / 2} \exp \left(2 \pi i \mathbf{y}^{T} \mathbf{q} a_{1}\right) I\left(2 \pi\left(w\left[a_{1}\right]\right)^{1 / 2}\left(\mathbf{y}^{T} W^{-1}\right)^{T}, n s / 2\right)$

where

$$
I(\mathbf{y}, s):=\int_{\mathbb{R}^{n-1}}\left(1+\mathbf{x}^{T} \mathbf{x}\right)^{-s} \exp \left(-i \mathbf{y}^{T} \mathbf{x}\right) d \mathbf{x}, \quad \operatorname{Re}(s)>\frac{n-1}{2} .
$$

We now evaluate $I(\mathbf{y}, s)$. For $\mathbf{y}=\mathbf{0}$, we have (see [10] p. 480-481)

$$
I(\mathbf{0}, s)=\pi^{(n-1) / 2} \frac{\Gamma(s-(n-1) / 2)}{\Gamma(s)} .
$$

For $\mathbf{y} \neq \mathbf{0}$, we follow ([10] p. 481). By ([9] Theorem 3.3, p. 155), we have

$$
I(\mathbf{y}, s)=(2 \pi)^{(n-1) / 2} \int_{0}^{\infty}\left(1+x^{2}\right)^{-s} x^{n-2} J_{\frac{n}{2}-\frac{3}{2}}(\| \mathbf{y}|| x)(\| \mathbf{y}|| x)^{\frac{3}{2}-\frac{n}{2}} d x
$$

where

$$
J_{\nu}(x):=(1 / 2 x)^{\nu} \pi^{-1 / 2} \Gamma(\mu+1 / 2)^{-1} \int_{0}^{\pi} \exp (-i x \cos (t)) \sin (t)^{2 v} d t, \quad \operatorname{Re}(v)>-\frac{1}{2}
$$

is the $J$-Bessel function of the first kind and $\|\mathbf{y}\|=\left(\mathbf{y}^{T} \mathbf{y}\right)^{1 / 2}$. Then by ([1] p. 488, eq. (11.4.44)), we have

$$
I(\mathbf{y}, s)=\frac{(2 \pi)^{(n-1) / 2}(\|\mathbf{y}\| / 2)^{s-(n-1) / 2}}{\Gamma(s)} K_{\frac{n-1}{2}-s}(\|\mathbf{y}\|)
$$

where

$$
K_{v}(z):=\frac{1}{2} \int_{0}^{\infty} \exp \left(-z\left(u+\frac{1}{u}\right) / 2\right) u^{v-1} d u, \quad|\arg (z)|<\frac{\pi}{2}
$$

is the modified $K$-Bessel function.

Apply the Poisson summation formula to the sum over $\mathbf{b} \in \mathbb{Z}^{n-1}$ in (10) to get

$$
\pi^{-n s / 2} \Gamma(n s / 2)\left(y_{1}^{n-1} y_{2}^{n-2} \cdots y_{n-1}\right)^{s} \sum_{\substack{a_{1} \in \mathbb{Z} \\ a_{1} \neq 0}} \sum_{\mathbf{b} \in \mathbb{Z}^{n-1}} \widehat{f}(\mathbf{b}, s) .
$$

To analyze this expression we separate the cases $\mathbf{b}=\mathbf{0}$ and $\mathbf{b} \neq \mathbf{0}$. If $\mathbf{b}=\mathbf{0}$, then (11) yields

$$
\begin{aligned}
\widehat{f}(\mathbf{0}, s) & =\left(w\left[a_{1}\right]\right)^{\frac{n-1}{2}-\frac{n s}{2}} \operatorname{Det}(S)^{-1 / 2} I(\mathbf{0}, n s / 2) \\
& =\operatorname{Det}(S)^{-1 / 2} \pi^{(n-1) / 2} \frac{\Gamma\left(\frac{n s}{2}-\frac{n-1}{2}\right)}{\Gamma(n s / 2)} w^{\frac{n-1}{2}-\frac{n s}{2}}\left|a_{1}\right|^{(n-1)-n s} .
\end{aligned}
$$

Hence the contribution of $\mathbf{b}=\mathbf{0}$ to (13) is

$$
2 \operatorname{Det}(S)^{-1 / 2}\left(y_{1}^{n-1} y_{2}^{n-2} \cdots y_{n-1}\right)^{s} \pi^{-\frac{n s}{2}+\frac{(n-1)}{2}} \Gamma\left(\frac{n s}{2}-\frac{n-1}{2}\right) w^{\frac{n-1}{2}-\frac{n s}{2}} \zeta(n s-(n-1)),
$$

where we used

$$
\zeta(n s-(n-1))=\frac{1}{2} \sum_{\substack{a_{1} \in \mathbb{Z} \\ a_{1} \neq 0}}\left|a_{1}\right|^{(n-1)-n s} .
$$


To compute the Laurent expansion of (14) at $s=1$, note that

$$
\begin{aligned}
&\left(y_{1}^{n-1} y_{2}^{n-2} \cdots y_{n-1}\right)^{s}= y_{1}^{n-1} y_{2}^{n-2} \cdots y_{n-1}+\left(y_{1}^{n-1} y_{2}^{n-2} \cdots y_{n-1}\right) \log \left(y_{1}^{n-1} y_{2}^{n-2} \cdots y_{n-1}\right)(s-1) \\
&+O\left(|s-1|^{2}\right), \\
& \pi^{-\frac{n s}{2}+\frac{(n-1)}{2}} \Gamma\left(\frac{n s}{2}-\frac{n-1}{2}\right)=1+\frac{n \gamma_{0}}{2}(s-1)+O\left(|s-1|^{2}\right)
\end{aligned}
$$

where $\gamma_{0}=-\gamma-\log (\pi)-2 \log (2)$ (here $\gamma$ is Euler's constant),

$$
w^{\frac{n-1}{2}-\frac{n s}{2}}=w^{-1 / 2}-\frac{n}{2} \log (w) w^{-1 / 2}(s-1)+O\left(|s-1|^{2}\right),
$$

and

$$
\zeta(n s-(n-1))=\frac{1 / n}{s-1}+\gamma+O(|s-1|) .
$$

Then multiplying terms yields the Laurent expansion

$$
\begin{aligned}
& \frac{\frac{2}{n}(\operatorname{Det}(S) w)^{-1 / 2}\left(y_{1}^{n-1} y_{2}^{n-2} \cdots y_{n-1}\right)}{s-1} \\
& \quad+2 \gamma(\operatorname{Det}(S) w)^{-1 / 2}\left(y_{1}^{n-1} y_{2}^{n-2} \cdots y_{n-1}\right) \\
& \quad+\frac{2}{n}(\operatorname{Det}(S) w)^{-1 / 2}\left(y_{1}^{n-1} y_{2}^{n-2} \cdots y_{n-1}\right)\left(\log \left(y_{1}^{n-1} y_{2}^{n-2} \cdots y_{n-1}\right)+\frac{n \gamma_{0}}{2}-\frac{n}{2} \log (w)\right) \\
& +O(|s-1|) \\
& \quad=\frac{2 / n}{s-1}+2 \gamma+\gamma_{0}+\frac{2}{n} \log \left(y_{1}^{n-1} y_{2}^{n-2} \cdots y_{n-1}\right)-\log (w)+O(|s-1|) \\
& \quad=\frac{2 / n}{s-1}+\gamma-\log (4 \pi)+\frac{2}{n} \log \left(y_{1}^{n-1} y_{2}^{n-2} \cdots y_{n-1}\right)-\log (w)+O(|s-1|)
\end{aligned}
$$

where we used

$$
\operatorname{Det}(S) w=\operatorname{Det}(Q)=\operatorname{Det}(Z)^{2}=\left(y_{1}^{n-1} y_{2}^{n-2} \cdots y_{n-1}\right)^{2} .
$$

We now calculate $w=m-\mathbf{q}^{T} S \mathbf{q}$. We have

$$
\mathbf{q}=S^{-1} \mathbf{r}=\left(\left(Z_{1}^{T}\right)^{-1} Z_{1}^{-1}\right) Z_{1}\left(\begin{array}{c}
x_{1,2} y_{1} y_{2} \cdots y_{n-2} \\
x_{1,3} y_{1} y_{2} \cdots y_{n-3} \\
\vdots \\
x_{1, n}
\end{array}\right)=\left(Z_{1}^{T}\right)^{-1}\left(\begin{array}{c}
x_{1,2} y_{1} y_{2} \cdots y_{n-2} \\
x_{1,3} y_{1} y_{2} \cdots y_{n-3} \\
\vdots \\
x_{1, n}
\end{array}\right) .
$$

Therefore

$$
\begin{aligned}
\mathbf{q}^{T} S \mathbf{q} & =\left(x_{1,2} y_{1} y_{2} \cdots y_{n-2}, x_{1,3} y_{1} y_{2} \cdots y_{n-3}, \cdots, x_{1, n}\right) Z_{1}^{-1}\left(Z_{1} Z_{1}^{T}\right)\left(Z_{1}^{T}\right)^{-1}\left(\begin{array}{c}
x_{1,2} y_{1} y_{2} \cdots y_{n-2} \\
x_{1,3} y_{1} y_{2} \cdots y_{n-3} \\
\vdots \\
x_{1, n}
\end{array}\right) \\
& =\left(x_{1,2} y_{1} y_{2} \cdots y_{n-2}\right)^{2}+\left(x_{1,3} y_{1} y_{2} \cdots y_{n-3}\right)^{2}+\cdots+x_{1, n}^{2},
\end{aligned}
$$

so that

$$
\begin{aligned}
w= & m-\mathbf{q}^{T} \mathbf{S} \mathbf{q} \\
= & \left(y_{1} y_{2}+\cdots y_{n-1}\right)^{2}+\left(x_{1,2} y_{1} y_{2} \cdots y_{n-2}\right)^{2}+\left(x_{1,3} y_{1} y_{2} \cdots y_{n-3}\right)^{2}+\cdots+x_{1, n}^{2} \\
& -\left(\left(x_{1,2} y_{1} y_{2} \cdots y_{n-2}\right)^{2}+\left(x_{1,3} y_{1} y_{2} \cdots y_{n-3}\right)^{2}+\cdots+x_{1, n}^{2}\right) \\
= & \left(y_{1} y_{2}+\cdots y_{n-1}\right)^{2} .
\end{aligned}
$$


Substituting this formula for $w$ into (15) and simplifying yields the Laurent expansion

$$
\frac{2 / n}{s-1}+\gamma-\log (4 \pi)-\frac{2}{n} \log \left(y_{1} y_{2}^{2} \cdots y_{n-1}^{n-1}\right)+O(|s-1|) .
$$

If $\mathbf{b} \neq \mathbf{0}$, then (12) yields

$$
\begin{gathered}
\widehat{f}(\mathbf{b}, s)=\left(w\left[a_{1}\right]\right)^{\frac{n-1}{2}-\frac{n s}{2}} \operatorname{Det}(S)^{-1 / 2} \exp \left(2 \pi i \mathbf{b}^{T} \mathbf{q} a_{1}\right) I\left(2 \pi\left(w\left[a_{1}\right]\right)^{1 / 2}\left(\mathbf{b}^{T} W^{-1}\right)^{T}, n s / 2\right) \\
=\left(w\left[a_{1}\right]\right)^{\frac{n-1}{2}-\frac{n s}{2}} \operatorname{Det}(S)^{-1 / 2} \exp \left(2 \pi i \mathbf{b}^{T} \mathbf{q} a_{1}\right) \\
\quad \times \frac{(2 \pi)^{(n-1) / 2}\left(\left\|2 \pi\left(w\left[a_{1}\right]\right)^{1 / 2}\left(\mathbf{b}^{T} W^{-1}\right)^{T}\right\| / 2\right)^{\frac{n s}{2}-\frac{n-1}{2}}}{\Gamma(n s / 2)} \\
=\left(w\left[a_{1}\right]\right)^{\frac{n-1}{2}-\frac{n s}{2}} \operatorname{Det}(S)^{-1 / 2} \exp \left(2 \pi i \mathbf{b}^{T} \mathbf{q} a_{1}\right) \\
\quad \times \frac{(2 \pi)^{(n-1) / 2}\left(\frac{1}{2} \cdot 2 \pi w^{1 / 2}\left(S^{-1}[\mathbf{b}]\right)^{1 / 2}\left|a_{1}\right|\right)^{\frac{n s}{2}-\frac{n-1}{2}}}{\Gamma(n s / 2)} \\
\times K_{\frac{n-1}{2}-\frac{n s}{2}}\left(2 \pi w^{1 / 2}\left(S^{-1}[\mathbf{b}]\right)^{1 / 2}\left|a_{1}\right|\right),
\end{gathered}
$$

where we used

$$
\left\|2 \pi\left(w\left[a_{1}\right]\right)^{1 / 2}\left(\mathbf{b}^{T} W^{-1}\right)^{T}\right\|=2 \pi\left(w\left[a_{1}\right] S^{-1}[\mathbf{b}]\right)^{1 / 2}=2 \pi w^{1 / 2}\left(S^{-1}[\mathbf{b}]\right)^{1 / 2}\left|a_{1}\right| .
$$

Now, using the functional equation $K_{-v}(z)=K_{v}(z)$ and the identity

$$
K_{1 / 2}(z)=(\pi / 2 z)^{1 / 2} e^{-z},
$$

we have

$$
\begin{aligned}
\widehat{f}(\mathbf{b}, 1)= & \left(w\left[a_{1}\right]\right)^{-1 / 2} \operatorname{Det}(S)^{-1 / 2} \exp \left(2 \pi i \mathbf{b}^{T} \mathbf{q} a_{1}\right) \\
& \times(2 \pi)^{(n-1) / 2} \frac{(\pi / 4)^{1 / 2}}{\Gamma(n / 2)} \exp \left(-2 \pi w^{1 / 2}\left(S^{-1}[\mathbf{b}]\right)^{1 / 2}\left|a_{1}\right|\right) \\
= & \left(y_{1}^{n-1} y_{2}^{n-2} \cdots y_{n-1}\right)^{-1}\left|a_{1}\right|^{-1} \frac{\pi^{n / 2}}{\Gamma(n / 2)} \exp \left(2 \pi i\left(\mathbf{b}^{T} \mathbf{q} a_{1}+i w^{1 / 2}\left(S^{-1}[\mathbf{b}]\right)^{1 / 2}\left|a_{1}\right|\right)\right),
\end{aligned}
$$

where we again used (16). Hence if $s=1$, the contribution of the terms with $\mathbf{b} \neq \mathbf{0}$ to (13) is

$$
\begin{aligned}
& \sum_{\substack{a_{1} \in \mathbb{Z} \\
a_{1} \neq 0}} \sum_{\substack{\mathbf{b} \in \mathbb{Z}^{n-1} \\
\mathbf{b} \neq \mathbf{0}}}\left|a_{1}\right|^{-1} \exp \left(2 \pi i\left(\mathbf{b}^{T} \mathbf{q} a_{1}+i w^{1 / 2}\left(S^{-1}[\mathbf{b}]\right)^{1 / 2}\left|a_{1}\right|\right)\right) \\
& =4 \sum_{a_{1}=1}^{\infty} \sum_{\substack{\left.\mathbf{b} \in \mathbb{Z}^{n-1} \\
\mathbf{b o d} \pm 1\right) \\
\mathbf{b} \neq \mathbf{0}}} \frac{1}{a_{1}} \operatorname{Re}\left(\exp \left(\left(-2 \pi w^{1 / 2}\left(S^{-1}[\mathbf{b}]\right)^{1 / 2}+2 \pi i \mathbf{b}^{T} \mathbf{q}\right) a_{1}\right)\right) \\
& =4 \sum_{\substack{\left.\mathbf{b} \in \mathbb{Z}^{n-1} \\
\mathbf{b o d} \pm 1\right) \\
\mathbf{b} \neq \mathbf{0}}}-\log \left|1-\exp \left(-2 \pi w^{1 / 2}\left(S^{-1}[\mathbf{b}]\right)^{1 / 2}+2 \pi i \mathbf{b}^{T} \mathbf{q}\right)\right| \\
& =-4 \log \prod_{\substack{\left.\mathbf{b} \in \mathbb{Z}^{n-1} \\
\mathbf{b} \bmod \pm 1\right)}}\left|1-\exp \left(-2 \pi w^{1 / 2}\left(S^{-1}[\mathbf{b}]\right)^{1 / 2}+2 \pi i \mathbf{b}^{T} \mathbf{q}\right)\right|,
\end{aligned}
$$


where we used

$$
\operatorname{Re}\left(\sum_{k=1}^{\infty} \frac{z^{k}}{k}\right)=-\log |1-z| .
$$

Finally, by combining (8), (17) and (18), we get the Laurent expansion

$$
E_{n}^{*}(Z, s)=\frac{2 / n}{s-1}+\gamma-\log (4 \pi)-\frac{2}{n} \log \left(y_{1} y_{2}^{2} \cdots y_{n-1}^{n-1}\right)-4 \log g(Z)+O(|s-1|),
$$

where

$$
\begin{aligned}
g(Z):= & \exp \left(-\frac{\left(y_{1} y_{2}^{2} \cdots y_{n-1}^{n-1}\right)^{1 /(n-1)} E_{n-1}^{*}\left(Z_{1}, n /(n-1)\right)}{4}\right) \\
& \times \prod_{\substack{\mathbf{b} \in \mathbb{Z}^{n-1} \\
\mathbf{b}\left(\bmod _{\mathbf{b} \neq \mathbf{0}} \pm 1\right)}}\left|1-\exp \left(-2 \pi w^{1 / 2}\left(S^{-1}[\mathbf{b}]\right)^{1 / 2}+2 \pi i \mathbf{b}^{T} \mathbf{q}\right)\right| .
\end{aligned}
$$

This proves Proposition 1.

\section{Dedekind zeta functions of totally real fields}

In this section we relate the zeta function of a wide ideal class of a totally real number field of degree $n$ to the integral of the maximal parabolic Eisenstein series $E_{n}^{*}(Z, s)$ along a Heegner cycle in $\mathcal{H}^{n}$ and thus prove Proposition 2.

Let $F$ be a totally real number field of degree $n$ and $U$ be the group of units of $F$. Let $A$ be a wide ideal class of $F$ and fix $\mathfrak{B} \in A^{-1}$. Then the ideal class zeta function may be written as

$$
\zeta_{F}(s, A):=\sum_{\substack{\mathfrak{A} \in A \\ \mathfrak{A} \neq 0}} \frac{1}{N(\mathfrak{A})^{s}}=N(\mathfrak{B})^{s} \sum_{\lambda \in \mathfrak{B}^{*} / U} \frac{1}{|N(\lambda)|^{\mid}}, \quad \operatorname{Re}(s)>1
$$

where $N(\mathfrak{A})$ is the norm and $\mathfrak{B}^{*}=\mathfrak{B} \backslash\{0\}$. Let $\lambda_{1}, \lambda_{2}, \ldots, \lambda_{n}$ denote the images of $\lambda \in \mathfrak{B}$ under the real embeddings of $F$. Then

$$
|N(\lambda)|^{s}=\left|\lambda_{1} \lambda_{2} \cdots \lambda_{n}\right|^{s}
$$

Note that for $x>0$,

$$
x^{-s}=\frac{1}{\Gamma(s / 2)} \int_{0}^{\infty} e^{-x^{2} t} t^{s / 2} \frac{d t}{t} .
$$

Then for $a_{1}, a_{2}, \ldots, a_{n}>0$,

$$
\begin{aligned}
& \left(a_{1} a_{2} \cdots a_{n}\right)^{-s} \Gamma(s / 2)^{n}= \\
& \int_{0}^{\infty} \int_{0}^{\infty} \cdots \int_{0}^{\infty} \exp \left(-\left(a_{1}^{2} t_{1}+a_{2}^{2} t_{2}+\cdots a_{n}^{2} t_{n}\right)\right)\left(t_{1} t_{2} \cdots t_{n}\right)^{s / 2} \frac{d t_{1}}{t_{1}} \frac{d t_{2}}{t_{2}} \cdots \frac{d t_{n}}{t_{n}}
\end{aligned}
$$

Consider the change of variables 


$$
\begin{aligned}
t_{1} & =x_{1}^{2} w \\
t_{2} & =x_{2}^{2} w \\
\vdots & \\
t_{n-1} & =x_{n-1}^{2} w \\
t_{n} & =\left(x_{1} x_{2} \cdots x_{n-1}\right)^{-2} w
\end{aligned}
$$

and the corresponding Jacobian

$$
J=n 2^{n-1}\left(x_{1} x_{2} \cdots x_{n-1}\right)^{-1} w^{n-1} .
$$

Then making this change of variables in (20) yields

$$
\begin{aligned}
& \left(a_{1} a_{2} \cdots a_{n}\right)^{-s} \Gamma(s / 2)^{n}= \\
& n 2^{n-1} \int_{0}^{\infty} \int_{0}^{\infty} \cdots \int_{0}^{\infty}\left(\int_{0}^{\infty} \exp \left(-\left(\sum_{k=1}^{n-1} a_{k}^{2} x_{k}^{2}+a_{n}^{2}\left(x_{1} \cdots x_{n-1}\right)^{-2}\right) w\right) w^{n s / 2} \frac{d w}{w}\right) \\
& \quad \times \frac{d x_{1}}{x_{1}} \frac{d x_{2}}{x_{2}} \cdots \frac{d x_{n-1}}{x_{n-1}} \\
& =n 2^{n-1} \Gamma(n s / 2) \int_{0}^{\infty} \int_{0}^{\infty} \cdots \int_{0}^{\infty}\left(\sum_{k=1}^{n-1} a_{k}^{2} x_{k}^{2}+a_{n}^{2}\left(x_{1} \cdots x_{n-1}\right)^{-2}\right)^{-n s / 2} \\
& \quad \times \frac{d x_{1}}{x_{1}} \frac{d x_{2}}{x_{2}} \cdots \frac{d x_{n-1}}{x_{n-1}}
\end{aligned}
$$

We now apply the identity (21) in (19) to get

$$
\begin{aligned}
& \zeta_{F}(s, A) \Gamma(s / 2)^{n}=n 2^{n-1} \Gamma(n s / 2) N(\mathfrak{B})^{s} \\
& \quad \times \sum_{\lambda \in \mathfrak{B}^{*} / U} \int_{0}^{\infty} \int_{0}^{\infty} \cdots \int_{0}^{\infty}\left(\sum_{k=1}^{n-1} \lambda_{k}^{2} t_{k}^{2}+\lambda_{n}^{2}\left(t_{1} \cdots t_{n-1}\right)^{-2}\right)^{-n s / 2} \frac{d t_{1}}{t_{1}} \frac{d t_{2}}{t_{2}} \cdots \frac{d t_{n-1}}{t_{n-1}} .
\end{aligned}
$$

Given a unit $\varepsilon \in U$, let $\varepsilon_{1}, \varepsilon_{2}, \ldots, \varepsilon_{n}$ denote the images of $\varepsilon$ under the real embeddings of $F$. There is an action of the unit group $U$ on $\mathbb{R}_{+}^{n-1}$ given by

$$
\begin{aligned}
\varepsilon: & \mathbb{R}_{+}^{n-1} \longrightarrow \mathbb{R}_{+}^{n-1}, \\
& \left(t_{1}, t_{2}, \ldots, t_{n-1}\right) \longmapsto\left(\left|\varepsilon_{1}\right| t_{1},\left|\varepsilon_{2}\right| t_{2}, \ldots,\left|\varepsilon_{n-1}\right| t_{n-1}\right) .
\end{aligned}
$$

Let $\mathbb{R}_{+}^{n-1} / U$ denote a fundamental domain for this action. Then using this action, (22) becomes

$$
\begin{aligned}
& \zeta_{F}(s, A) \Gamma(s / 2)^{n}=n 2^{n-1} \Gamma(n s / 2) N(\mathfrak{B})^{s} \\
& \quad \times \sum_{\lambda \in \mathfrak{B}^{*}} \int_{\mathbb{R}_{+}^{n-1} / U} \ldots \int\left(\sum_{k=1}^{n-1} \lambda_{k}^{2} t_{k}^{2}+\lambda_{n}^{2}\left(t_{1} \cdots t_{n-1}\right)^{-2}\right)^{-n s / 2} \frac{d t_{1}}{t_{1}} \cdots \frac{d t_{n-1}}{t_{n-1}} .
\end{aligned}
$$

Let $\alpha_{1}, \alpha_{2}, \ldots, \alpha_{n}$ be a $\mathbb{Z}$-basis for $\mathfrak{B}$ and $\alpha_{1}^{(i)}, \alpha_{2}^{(i)}, \ldots, \alpha_{n}^{(i)}$ for $i=1,2, \ldots, n$ denote their images under the real embeddings of $F$. Given $\lambda \in \mathfrak{B}$, write

$$
\lambda=m_{1} \alpha_{1}+m_{2} \alpha_{2}+\cdots+m_{n} \alpha_{n}
$$


where $m_{1}, m_{2}, \ldots, m_{n} \in \mathbb{Z}$. Then

$$
\sum_{k=1}^{n-1} \lambda_{k}^{2} t_{k}^{2}+\lambda_{n}^{2}\left(t_{1} \cdots t_{n-1}\right)^{-2}=\mathbf{m}^{T}\left(M_{\mathfrak{B}}(\mathbf{t}) M_{\mathfrak{B}}(\mathbf{t})^{T}\right) \mathbf{m}
$$

where

$$
\mathbf{m}=\left(\begin{array}{l}
m_{1} \\
m_{2} \\
\vdots \\
m_{n}
\end{array}\right)
$$

and

$$
M_{\mathfrak{B}}(\mathbf{t}):=\left(\begin{array}{ccccc}
\alpha_{1}^{(1)} t_{1} & \alpha_{1}^{(2)} t_{2} & \cdots & \alpha_{1}^{(n-1)} t_{n-1} & \alpha_{1}^{(n)}\left(t_{1} t_{2} \cdots t_{n-1}\right)^{-1} \\
\alpha_{2}^{(1)} t_{1} & \alpha_{2}^{(2)} t_{2} & \cdots & \alpha_{2}^{(n-1)} t_{n-1} & \alpha_{2}^{(n)}\left(t_{1} t_{2} \cdots t_{n-1}\right)^{-1} \\
\vdots & \vdots & & \vdots & \vdots \\
\alpha_{n}^{(1)} t_{1} & \alpha_{n}^{(2)} t_{2} & \cdots & \alpha_{n}^{(n-1)} t_{n-1} & \alpha_{n}^{(n)}\left(t_{1} t_{2} \cdots t_{n-1}\right)^{-1}
\end{array}\right)
$$

where $\mathbf{t}=\left(t_{1}, t_{2}, \ldots, t_{n-1}\right) \in \mathbb{R}_{+}^{n-1}$.

Define

$$
Q_{\mathfrak{B}}(\mathbf{t}):=M_{\mathfrak{B}}(\mathbf{t}) M_{\mathfrak{B}}(\mathbf{t})^{T},
$$

and let

$$
Q_{\mathfrak{B}}(\mathbf{t})[\mathbf{m}]:=\mathbf{m}^{T} \cdot Q_{\mathfrak{B}}(\mathbf{t}) \cdot \mathbf{m}
$$

be the quadratic form associated to $Q_{\mathfrak{B}}(\mathbf{t})$. Then the identity (23) becomes

$$
\zeta_{F}(s, A) \Gamma(s / 2)^{n}=n 2^{n-1} \Gamma(n s / 2) N(\mathfrak{B})^{s} \int_{\mathbb{R}_{+}^{n-1} / U} \ldots \int_{/ U} \zeta\left(n s / 2, Q_{\mathfrak{B}}(\mathbf{t})\right) \frac{d t_{1}}{t_{1}} \cdots \frac{d t_{n-1}}{t_{n-1}},
$$

where

$$
\zeta\left(s, Q_{\mathfrak{B}}(\mathbf{t})\right):=\sum_{\substack{\mathbf{m} \in \mathbb{Z}^{n} \\ \mathbf{m} \neq \mathbf{0}}} Q_{\mathfrak{B}}(\mathbf{t})[\mathbf{m}]^{-s}, \quad \operatorname{Re}(s)>\frac{n}{2}
$$

is the Epstein zeta function of $Q_{\mathfrak{B}}(\mathbf{t})$.

The positive definite, symmetric matrix $Q_{\mathfrak{B}}(\mathbf{t})$ may be written as

$$
Q_{\mathfrak{B}}(\mathbf{t})=\operatorname{Det}\left(Q_{\mathfrak{B}}(\mathbf{t})\right)^{1 / n}\left(y_{1}^{n-1}(\mathbf{t}) y_{2}^{n-2}(\mathbf{t}) \cdots y_{n-1}(\mathbf{t})\right)^{-2 / n} \tau_{\mathfrak{B}}(\mathbf{t}) \tau_{\mathfrak{B}}(\mathbf{t})^{T}
$$

where

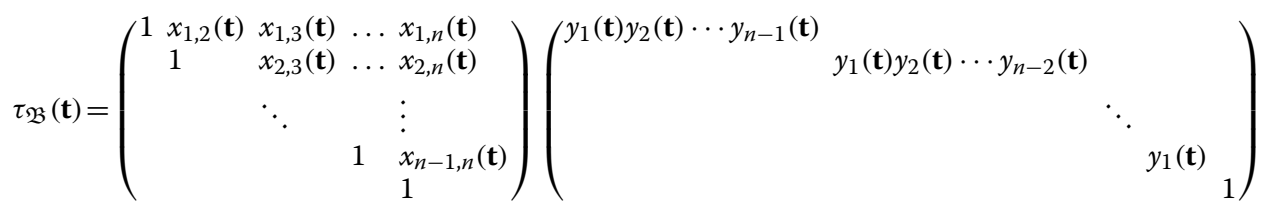

is in $\mathcal{H}^{n}$. Here we have suppressed the dependence of the variables $x_{i, j}(\mathbf{t})$ and $y_{i}(\mathbf{t})$ on $\mathfrak{B}$ and the $\mathbb{Z}$-basis $\alpha_{1}, \alpha_{2}, \ldots, \alpha_{n}$. Then $\left\{\tau_{\mathfrak{B}}(\mathbf{t}): \mathbf{t} \in \mathbb{R}_{+}^{n-1} / U\right\}$ defines a Heegner cycle in $\mathcal{H}^{n}$.

Now, by (6) we have the identity

$$
\zeta\left(n s / 2, Q_{\mathfrak{B}}(\mathbf{t})\right)=\operatorname{Det}\left(Q_{\mathfrak{B}}(\mathbf{t})\right)^{-s / 2} \zeta(n s) E_{n}\left(\tau_{\mathfrak{B}}(\mathbf{t}), s\right) .
$$


Moreover,

$$
\begin{aligned}
\operatorname{Det}\left(Q_{\mathfrak{B}}(\mathbf{t})\right) & =\operatorname{Det}\left(\begin{array}{cccc}
\alpha_{1}^{(1)} & \alpha_{1}^{(2)} & \cdots & \alpha_{1}^{(n)} \\
\alpha_{2}^{(1)} & \alpha_{2}^{(2)} & \cdots & \alpha_{2}^{(n)} \\
\vdots & \vdots & & \vdots \\
\alpha_{n}^{(1)} & \alpha_{n}^{(2)} & \cdots & \alpha_{n}^{(n)}
\end{array}\right)^{2} \\
& =\operatorname{disc}(\mathfrak{B}) \\
& =N(\mathfrak{B})^{2} D_{F}
\end{aligned}
$$

where $D_{F}$ is the discriminant of $F$. Then if

$$
\zeta_{F}^{*}(s, A):=\pi^{-n s / 2} \Gamma(s / 2)^{n} D_{F}^{s / 2} \zeta_{F}(s, A)
$$

denotes the completed ideal class zeta function, (24) yields

$$
\zeta_{F}^{*}(s, A)=n 2^{n-1} \int_{\mathbb{R}_{+}^{n-1} / U} \ldots \int_{n} E_{n}^{*}\left(\tau_{\mathfrak{B}}(\mathbf{t}), s\right) \frac{d t_{1}}{t_{1}} \cdots \frac{d t_{n-1}}{t_{n-1}} .
$$

This proves Proposition 2.

\section{Proof of Theorem 2}

Given a number field $K$, let $\mathrm{CL}(K)$ be the wide ideal class group, $h_{K}$ be the class number, $R_{K}$ be the regulator, $w_{K}$ be the number of roots of unity, and $D_{K}$ be the absolute value of the discriminant. Given an ideal class group character $\chi$ of $K$, the class group $L$-function is defined by

$$
L_{K}(\chi, s):=\sum_{A \in \mathrm{CL}(K)} \chi(A) \zeta_{K}(s, A), \quad \operatorname{Re}(s)>1
$$

where $\zeta_{K}(s, A)$ denotes the ideal class zeta function of $A \in \mathrm{CL}(K)$.

If $\chi$ is trivial, then $L(\chi, s)=\zeta_{K}(s)$ is the Dedekind zeta function of $K$. The Dedekind zeta function $\zeta_{K}(s)$ extends to a meromorphic function on $\mathbb{C}$ with a simple pole at $s=1$ with residue

$$
\operatorname{Res}_{s=1} \zeta_{K}(s)=\frac{2^{r_{1}}(2 \pi)^{r_{2}} h_{K} R_{K}}{w_{K} \sqrt{D_{K}}}
$$

where $r_{1}$ (resp. 2r $r_{2}$ ) is the number of real (resp. complex) embeddings of $K$.

Suppose now that $F$ is a totally real number field of degree $n$ and $H$ is the Hilbert class field of $F$. By class field theory, one has the factorization

$$
\frac{\zeta_{H}(s)}{\zeta_{F}(s)}=\prod_{\substack{\chi \in \overline{\mathrm{CL}(F)} \\ \chi \neq 1}} L_{F}(\chi, s) .
$$

Since $F$ is totally real of degree $n$ and $H$ is unramified at the infinite primes, $H$ is totally real with $n \cdot h_{F}$ real embeddings. It follows from (25) that

$$
\lim _{s \rightarrow 1} \frac{(s-1)}{(s-1)} \frac{\zeta_{H}(s)}{\zeta_{F}(s)}=2^{n\left(h_{F}-1\right)} \frac{h_{H}}{h_{F}} \frac{R_{H}}{R_{F}} \sqrt{\frac{D_{F}}{D_{H}}} .
$$

Here we used $w_{F}=w_{H}=2$, since these fields are totally real and hence have only the roots of unity \pm 1 . On the other hand, by Theorem 1 and orthogonality, for $\chi \neq 1$ we have

$$
L_{F}(\chi, 1)=-\frac{n 2^{n-1}}{D_{F}^{1 / 2}} \sum_{A \in \mathrm{CL}(F)} \chi(A) \rho_{n}(A)
$$


where we recall that

$$
\rho_{n}(A):=\int_{\mathbb{R}_{+}^{n-1} / U} \ldots \int_{\log } \log \left(\tau_{\mathfrak{B}}(\mathbf{t})\right) \frac{d t_{1}}{t_{1}} \cdots \frac{d t_{n-1}}{t_{n-1}}
$$

and $\mathfrak{B} \in A^{-1}$. Then combining (26) and (27) yields the identity

$$
\frac{(-1)^{h_{F}-1} 2^{h_{F}-1}}{n^{h_{F}-1}} \frac{h_{H}}{h_{F}} \frac{R_{H}}{R_{F}} \frac{D_{F}^{h_{F} / 2}}{D_{H}^{1 / 2}}=\prod_{\substack{x \in \overline{\mathrm{CL}(F)} \\ \chi \neq 1}} \sum_{A \in \mathrm{CL}(F)} \chi(A) \rho_{n}(A) .
$$

Since $D_{H}=D_{F}^{h_{F}}$, we have $D_{F}^{h_{F} / 2} / D_{H}^{1 / 2}=1$. Moreover, by a well-known result of Frobenius on group determinants (see e.g. [8] p. 78), we have

$$
\prod_{\substack{\chi \in \overline{\operatorname{CL}(F)} \\ \chi \neq 1}} \sum_{A \in \operatorname{CL}(F)} \chi(A) \rho_{n}(A)=\operatorname{Det}\left(\rho_{n}\left(A_{k}^{-1} A_{\ell}\right)-\rho_{n}\left(A_{k}^{-1}\right)\right)_{k, \ell}
$$

where $1 \leq k, \ell \leq h_{F}-1$. It follows that

$$
\frac{(-1)^{h_{F}-1} 2^{h_{F}-1}}{n^{h_{F}-1}} \frac{h_{H}}{h_{F}} \frac{R_{H}}{R_{F}}=\operatorname{Det}\left(\rho_{n}\left(A_{k}^{-1} A_{\ell}\right)-\rho_{n}\left(A_{k}^{-1}\right)\right)_{k, \ell} .
$$

Finally, if we write the ideal class group of $F$ as

$$
\mathrm{CL}(F)=\left\{A_{1}=\left[\mathfrak{A}_{1}\right]=\left[\mathcal{O}_{F}\right], A_{2}=\left[\mathfrak{A}_{2}\right], \ldots, A_{h_{F}}=\left[\mathfrak{A}_{h_{F}}\right]\right\},
$$

then

$$
\rho_{n}\left(A_{k}^{-1} A_{\ell}\right)-\rho_{n}\left(A_{k}^{-1}\right)=\int_{\mathbb{R}_{+}^{n-1} / U} \ldots \int_{U} \log \left(\frac{G\left(\tau_{\mathfrak{A}_{\ell}^{-1} \mathfrak{A}_{k}}(\mathbf{t})\right)}{G\left(\tau_{\mathfrak{A}_{\ell}}(\mathbf{t})\right)}\right) \frac{d t_{1}}{t_{1}} \cdots \frac{d t_{n-1}}{t_{n-1}} .
$$

This proves Theorem 2 .

\section{Proof of Theorem 3}

Let $F$ be a totally real abelian number field with $\operatorname{Gal}(F / \mathbb{Q}) \cong(\mathbb{Z} / 2 \mathbb{Z})^{\ell}$, and let $E$ be an unramified real quadratic extension of $F$ with $\operatorname{Gal}(E / \mathbb{Q}) \cong(\mathbb{Z} / 2 \mathbb{Z})^{\ell+1}$. Then the zeta function $\zeta_{E}(s)\left(\right.$ resp. $\left.\zeta_{F}(s)\right)$ factors as $\zeta(s)$ times the product of the Dirichlet $L$-functions associated to the quadratic subfields of $E$ (resp. $F$ ). Note that there are $2^{\ell}-1$ quadratic subfields of $F, 2^{\ell+1}-1$ quadratic subfields of $E$, and $2^{\ell}$ quadratic subfields of $E$ that are not contained in $F$. By class field theory, the unramified quadratic extension $E / F$ gives rise to a real ideal class group character $\chi_{E / F}$ of $F$ (a genus character) whose $L$-function factors as

$$
L_{F}\left(\chi_{E / F}, s\right)=\frac{\zeta_{E}(s)}{\zeta_{F}(s)} .
$$

Then by the preceding facts we obtain the factorization

$$
L_{F}\left(\chi_{E / F}, s\right)=\prod_{i=1}^{2^{\ell}} L\left(\chi_{i}, s\right)
$$

where $\chi_{i}$ for $1 \leq i \leq 2^{\ell}$ are the Kronecker symbols associated to the quadratic subfields $K_{i}$ of $E$ which are not contained in $F$.

By Dirichlet's class number formula, we have

$$
L\left(\chi_{i}, 1\right)=\frac{2 \log \left(\varepsilon_{i}\right) h_{i}}{\sqrt{\Delta_{i}}}
$$


where $\Delta_{i}>0, \varepsilon_{i}$ and $h_{i}$ are the discriminant, fundamental unit, and class number of $K_{i}$, resp. Therefore

$$
L_{F}\left(\chi_{E / F}, 1\right)=2^{2^{\ell}} \prod_{i=1}^{2^{\ell}} \frac{\log \left(\varepsilon_{i}\right) h_{i}}{\sqrt{\Delta_{i}}} .
$$

Let $n=2^{\ell}$ in (27) and equate this with (28) to get

$$
\sum_{A \in \mathrm{CL}(F)} \chi_{E / F}(A) \rho_{2^{\ell}}(A)=-\frac{D_{F}^{1 / 2}}{2^{\ell-1}} \prod_{i=1}^{2^{\ell}} \frac{\log \left(\varepsilon_{i}\right) h_{i}}{\sqrt{\Delta_{i}}} .
$$

This proves Theorem 3 .

\section{Proof of Corollary 1}

Conjecture 1 asserts that if $\lambda_{1}, \lambda_{2}, \ldots, \lambda_{k}$ are $\mathbb{Q}$-linearly independent elements of

$$
\mathcal{L}:=\left\{\log (\alpha): \alpha \in \overline{\mathbb{Q}}^{*}\right\},
$$

then $\lambda_{1}, \lambda_{2}, \ldots, \lambda_{k}$ are algebraically independent over $\mathbb{Q}$ (recall that algebraic independence over $\mathbb{Q}$ means that if $P\left(X_{1}, X_{2}, \ldots, X_{k}\right)$ is a nonzero polynomial with coefficients in $\mathbb{Q}$, then $\left.P\left(\lambda_{1}, \lambda_{2}, \ldots, \lambda_{k}\right) \neq 0\right)$. It follows that if $R\left(X_{1}, X_{2}, \ldots, X_{k}\right)$ is a nonconstant polynomial with coefficients in $\overline{\mathbb{Q}}$, then the number $R\left(\lambda_{1}, \lambda_{2}, \ldots, \lambda_{k}\right)$ is transcendental.

Now, because the units $\varepsilon_{i}, 1 \leq i \leq 2^{\ell}$, are multiplicatively independent, the numbers $\log \left(\varepsilon_{i}\right), 1 \leq i \leq 2^{\ell}$, are $\mathbb{Q}$-linearly independent. Define the (nonconstant) polynomial

$$
R\left(X_{1}, X_{2}, \ldots, X_{2^{\ell}}\right):=\frac{D_{F}^{1 / 2}}{2^{\ell-1}} \prod_{i=1}^{2^{\ell}} \alpha_{i} X_{i} \in \overline{\mathbb{Q}}\left[X_{1}, X_{2}, \ldots, X_{2^{\ell}}\right]
$$

where

$$
\alpha_{i}:=\frac{h_{i}}{\sqrt{\Delta_{i}}} .
$$

Then assuming Conjecture 1 , the number

$$
R\left(\log \left(\varepsilon_{1}\right), \log \left(\varepsilon_{2}\right), \ldots, \log \left(\varepsilon_{2} \ell\right)\right)
$$

is transcendental. However, by Theorem 3 we have

$$
R\left(\log \left(\varepsilon_{1}\right), \log \left(\varepsilon_{2}\right), \ldots, \log \left(\varepsilon_{2^{\ell}}\right)\right)=\sum_{A \in \mathrm{CL}(F)} \chi_{E / F}(A) \rho_{2^{\ell}}(A) .
$$

This proves Corollary 1.

\section{Acknowledgements}

We would like to thank Adrian Barquero-Sanchez for help computing the examples in Section 2 using SAGE. We would also like to thank the referee for helpful comments which improved the exposition of the paper. The second author was supported by the NSF grant DMS-1162535 during the preparation of this work.

\section{Author details}

${ }^{1}$ Department of Mathematics, Washington State University, Pullman 99164-3113, WA, USA. ${ }^{2}$ Department of Mathematics, Texas A\&M University, Mailstop 3368, College Station, 77843-3368 TX, USA.

Received: 21 April 2015 Accepted: 26 April 2015

Published online: 01 September 2015

\section{References}

1. Abramowitz, M, Stegun IA: Handbook of mathematical functions with formulas, graphs, and mathematical tables. Nat. Bureau Stand. Appl. Math. Ser. 55. For sale by the Superintendent of Documents, U.S. Government Printing Office, Washington, D.C. (1964) xiv+1046 pp

2. Bump, D, Goldfeld, D: A Kronecker limit formula for cubic fields. Modular forms (Durham, 1983), 43-49, Ellis Horwood Ser. Math. Appl.: Statist. Oper. Res., Horwood, Chichester (1984) 
3. Costa, A, Friedman, E: Ratios of regulators in totally real extensions of number fields. J. Number Theory. 37, 288-297 (1991)

4. Efrat, I: On a $G L(3)$ analog of $|\eta(z)|$. J. Number Theory. 40, 174-186 (1992)

5. Friedberg, S: A global approach to the Rankin-Selberg convolution for $G L(3, \mathbb{Z})$. Trans. Amer. Math. Soc. $\mathbf{3 0 0}$ 159-174 (1987)

6. Goldfeld, D: Automorphic forms and $L$-functions for the group $G L(n, \mathbb{R})$. With an appendix by Kevin A. Broughan. Cambridge Studies in Advanced Mathematics, Vol. 99. Cambridge University Press, Cambridge (2006). xiv+493

7. Kudla, S: Seesaw dual reductive pairs. Automorphic forms of several variables (Katata, 1983), 244-268. Progr. Math., 46, BirkhÃd' user Boston, Boston, MA (1984)

8. Siegel, CL: Advanced analytic number theory. Second edition. Tata Institute of Fundamental Research Studies in Mathematics, 9. Tata Institute of Fundamental Research, Bombay (1980). v+268

9. Stein, EM, Weiss, G: Introduction to Fourier analysis on Euclidean spaces. Princeton Mathematical Series, No. 32. Princeton University Press, Princeton, N.J. (1971). x+297

10. Terras, A: Bessel series expansions of the Epstein zeta function and the functional equation. Trans. Amer. Math. Soc 183, 477-486 (1973)

11. Waldschmidt, M: Diophantine approximation on linear algebraic groups. Transcendence properties of the exponential function in several variables. Grundlehren der Mathematischen Wissenschaften [Fundamental Principles of Mathematical Sciences], 326. Springer-Verlag, Berlin (2000). xxiv+633

12. Yue, Q: Genus fields of real biquadratic fields. Ramanujan J. 21, 17-25 (2010)

\section{Submit your manuscript to a SpringerOpen ${ }^{\odot}$ journal and benefit from:}

- Convenient online submission

- Rigorous peer review

- Immediate publication on acceptance

- Open access: articles freely available online

- High visibility within the field

Retaining the copyright to your article

Submit your next manuscript at $\boldsymbol{\triangleright}$ springeropen.com 